\title{
Balking and Re-service in a Vacation Queue with Batch Arrival and Two Types of Heterogeneous Service
}

\author{
Monita Baruah ${ }^{1}$, Kailash C. Madan $^{2} \&$ Tillal Eldabi ${ }^{3}$ \\ ${ }^{1}$ Brunel University, UK \\ ${ }^{2}$ College of Information Technology, Ahlia University, Kingdom of Bahrain \\ ${ }^{3}$ Brunel Business School, Brunel University, UK \\ Correspondence: Monita Baruah, Department of Mathematics, University of Bahrain, Kingdom of Bahrain. \\ E-mail: Monita.Baruah@brunel.ac.uk, monitabrh@gmail.com
}

Received: June 17, 2012 Accepted: July 2, 2012 Online Published: July 26, 2012

doi:10.5539/jmr.v4n4p114 URL: http://dx.doi.org/10.5539/jmr.v4n4p114

\begin{abstract}
In this paper we have considered a single server queue with customers or units arriving in groups or batches in a system providing two types of general heterogeneous service. The server provides type 1 or type 2 services on a first come first served basis. At the beginning of a service, a customer has the option to choose either type 1 or type 2 service. Also we added the concept of balking and re-service in this study. Balking is a kind of customer behavior. If a batch on arrival for service is reluctant or refuses to join the system for some reasons is said to balk. Once a service is completed the customer may leave the system or he has the option to demand re-service for the same service taken. Further as soon as service of any type gets completed, the server may take vacation or continue staying at the system even if the system is empty. For this model we have derived the steady state queue size distribution at random epoch and some particular cases have been developed and compared with known results.
\end{abstract}

Keywords: batch arrivals, general heterogeneous service, balking, vacations, queue size distribution, waiting time

\section{Introduction}

Research studies on queues with batch arrival and vacations have been increased tremendously and still many researchers have been developing on the theory of different aspects of queuing. Queuing is an important phenomenon in the real life, be it human or virtual queues and eminent researchers have been developing theories on queuing models since 1950. Research authors like Baba (1986), Lee et al. (1994; 1995), Doshi (1986) and Takagi (1990) were the pioneers in this field. We can find vast literature on the extensive amount of studies been done on different queuing models with server vacations, arrival pattern and customers behavior. Studies on queues with vacations and breakdown had been done by authors like Borthakur and Choudhury (1997; 2000; 2004), Madan (1995; 2000; 2001), Madan and Dayyeh (2002), Choudhury and Madan (2007). Many of the authors here have studied single server with different vacation policies. Queues with vacations and restricted admissibility of customers have been studied by Choudhury (2007) and Madan (2000).

In our model we assume customers arriving in batches and the system is providing in parallel two types of general heterogeneous service, the customer can choose any one type of service. We can find many real life applications of this model. Such situations are mostly observed in fuel stations, banks, post offices etc. where the customer has option of choosing any one kind of service.

The concept of balking was first studied by Haight in the year 1957, since then considerable attention has been given on many queuing theories with customer impatience. In our model we assume that arrival units may refuse to join the system (balks) by estimating the duration of waiting time for a service to get completed or by witnessing the long length of the queue. In queuing literature, queues with vacations and behavior of impatient customers have been studied by many for mainly single server but balking in case of two parallel servers for a general distribution has still not been worked on.

Re-service is also an important factor in the theory of queuing system and have many applications in the real world. There may be situations where re-service is desired, for instance while visiting a doctor, the patient may be 
recommended for some investigations, after which he may need to see the doctor again while in some situations offering public service a customer may find his service unsatisfactory and consequently demand re-service. We find many real life applications of queues with balking and re-service in sectors as telecommunication, computer networking, call centers, inventory and production, maintenance and quality control in industrial organisations etc. Though in the queuing literature we find limited studies on queuing system with re-service, we refer to some type of queuing networks including cyclic queuing systems with feedback studied by authors like Glenbe and Pujolle (1987) and Madan (1988). In fact, authors like Madan et al. (2004), Jeyakumar and Arumuganathan (2011) studied queues with re-service. Here we have discussed the case of optional re-service. In this case a customer has the choice of selecting any one of the two types of heterogeneous service, subsequently has the option to repeat the service taken by him or depart from the system.

The mathematical model has been defined under the following assumptions.

\section{Mathematical Model}

Here we assume that customers (units) arrive in batches of variable size according following a compound Poisson Process.

Let $\lambda c_{i} d t(i=1,2,3, \ldots)$ be the first order probability of arrival of ' $\mathrm{i}$ ' customers in batches in the system at a short interval of time $(t, t+d t]$ where $0 \leq c_{i} \leq 1, \sum_{i=1}^{\infty} c_{i}=1, \lambda>0$ is the mean arrival rate of batches.

We consider the case when there is a single server providing parallel service of two types on a first come first served basis (FCFS). At the start of the service, each customer has the choice of choosing either first service with probability $\xi_{1}$ or can choose second service with probability $\xi_{2}$ and $\xi_{1}+\xi_{2}=1$.

We assume that the random variable of service time $s_{j}(j=1,2)$ of the $j$ th kind of service follows a general probability law with distribution function $H_{j}\left(s_{j}\right), h_{j}\left(s_{j}\right)$ is the probability density function and $E\left(s_{j}^{K}\right)$ is the $k$ th moment $(k=1,2, \ldots)$ of service time, $j=1,2$.

Let $\mu_{j}(x)$ be the conditional probability of type $j$ service during the period $(x, x+d x]$ given that elapsed time is $x$, so that

$$
\mu_{j}(x)=\frac{h_{j}(x)}{1-H_{j}(x)}, j=1,2
$$

and

$$
h_{j}(x)=\mu_{j}\left(s_{j}\right) \exp \left[-\int_{0}^{s} \mu_{j}(x) d x\right], j=1,2 .
$$

Once the service of a customer is complete, the server may decide to take vacation with probability $q$ or may continue to serve the next customer with probability $(1-q)$ or may remain idle in the system even if there is no customer requiring service.

Further we assume that the vacation time random variable follow general probability law with distribution function $V(x), v(x)$ the probability density and $E\left(V^{K}\right)$ as the $k$ th moment, $k=1,2, \ldots$

Let $\phi(x)$ be the conditional probability of a vacation period during the interval $(x, x+d x]$ given elapsed time is $x$, so that

$$
\phi(x)=\frac{V(x)}{1-V(x)}
$$

and

$$
v(y)=\phi(y) \exp \left[-\int_{0}^{v} \phi(x) d x\right]
$$

Also we assume that $\left(1-a_{1}\right)\left(0 \leq a_{1} \leq 1\right)$ is the probability that an arriving batch balks during the period when the server is busy (available on the system) and $\left(1-a_{2}\right)\left(0 \leq a_{2} \leq 1\right)$ is the probability that an arriving batch balks during the period when server is on vacation.

As soon as service (of any one kind) is complete he has the option to leave the system or join the system for reservice, if necessary. We assume that probability of repeating type $j$ service as $r_{j}$ and leaving the system without re-service as $\left(1-r_{j}\right), j=1,2$. We consider that either service maybe repeated only once.

\section{Definitions and Notations}

Assuming that steady state exists, we define 
$P_{n, j}(x)=$ Probability that there are $n(\geq 1)$ customers in the system including one customer in type $j$ service, $j=1,2$ and elapsed service time is $x$.

Thus $P_{n, j}=\int_{0}^{\infty} P_{n, j}(x) d x$ is the corresponding steady state probability irrespective of elapsed time $x$.

$R_{n, j}(x)=$ Probability that there are $n(\geq 1)$ customers in the system including one customer who is repeating type $j$ service, $j=1,2$ and elapsed service time is $x$.

Thus $R_{n, j}=\int_{0}^{\infty} R_{n, j}(x) d x$ is the corresponding steady state probability irrespective of elapsed service time $x$.

$W_{n}(x)=$ probability that there are $n(\geq 0)$ customers in the queue and server is on vacation and elapsed vacation time is $x$. $W_{n}=\int_{0}^{\infty} W_{n}(x) d x$ is the corresponding steady state probability irrespective of elapsed vacation time $x$.

$E$ = Steady state probability of the server is idle but available in the system and there is no customer requiring service.

The Probability Generating Functions are defined as:

$$
\begin{gathered}
P_{j}(x, z)=\sum_{n=1}^{\infty} z^{n} P_{n, j}(x) ; P_{j}(z)=\sum_{n=1}^{\infty} z^{n} P_{n, j},|z| \leq 1 ; j=1,2 \\
R_{j}(x, z)=\sum_{n=1}^{\infty} z^{n} R_{n, j}(x) ; R_{j}(z)=\sum_{n=1}^{\infty} z^{n} R_{n, j} ;|z| \leq 1 ; j=1,2 \\
W(x, z)=\sum_{n=0}^{\infty} z^{n} W_{n}(x) ; W(z)=\sum_{n=0}^{\infty} z^{n} W_{n} ;|z| \leq 1 \\
C(z)=\sum_{i=1}^{\infty} z^{i} c_{i} ;|z| \leq 1
\end{gathered}
$$

\section{Equations Governing the System}

Let us define the steady state equations for our model as

$$
\begin{gathered}
\frac{d}{d x} P_{n, 1}(x)+\left(\lambda+\mu_{1}(x)\right) P_{n, 1}(x)=\lambda\left(1-a_{1}\right) P_{n, 1}(x)+a_{1} \lambda \sum_{i=1}^{n} c_{i} P_{n-i, 1}(x) \\
\frac{d}{d x} P_{n, 2}(x)+\left(\lambda+\mu_{2}(x)\right) P_{n, 2}(x)=\lambda\left(1-a_{1}\right) P_{n, 2}(x)+a_{1} \lambda \sum_{i=1}^{n} c_{i} P_{n-i, 2}(x) \\
\frac{d}{d x} R_{n, 1}(x)+\left(\lambda+\mu_{1}(x)\right) R_{n, 1}(x)=\lambda\left(1-a_{1}\right) R_{n, 1}(x)+a_{1} \lambda \sum_{i=1}^{\infty} c_{i} R_{n-i, 1}(x) \\
\frac{d}{d x} R_{n, 2}(x)+\left(\lambda+\mu_{2}(x)\right) R_{n, 2}(x)=\lambda\left(1-a_{1}\right) R_{n, 2}(x)+a_{1} \lambda \sum_{i=1}^{\infty} c_{i} R_{n-i, 2}(x) \\
\frac{d}{d x} W_{n}(x)+(\lambda+\phi(x)) W_{n}(x)=\lambda\left(1-a_{2}\right) W_{n}(x)+a_{2} \lambda \sum_{i=1}^{n} C_{i} W_{n-i}(x) \\
\frac{d}{d x} W_{0}(x)+(\lambda+\phi(x)) W_{0}(x)=\lambda\left(1-a_{2}\right) W_{0}(x) \\
\lambda E=\lambda\left(1-a_{1}\right) E+(1-q)\left[\left(1-r_{1}\right) \int_{0}^{\infty} P_{1,1}(x) \mu_{1}(x) d x+\left(1-r_{2}\right) \int_{0}^{\infty} P_{1,2}(x) \mu_{2}(x) d x\right] \\
+(1-q)\left[\int_{0}^{\infty} R_{1,1}(x) \mu_{1}(x) d x+\int_{0}^{\infty} R_{1,2}(x) \mu_{2}(x) d x\right]+\int_{0}^{\infty} W_{0}(x) \phi(x) d x
\end{gathered}
$$

where $P_{0, j}(x)=0, R_{0, j}(x)=0, j=1,2$ for (9), (10), (11) and (12).

The above equations is to be solved subject to the boundary conditions given below at $x=0$ :

$$
P_{n, 1}(0)=(1-q) \xi_{1}\left[\left(1-r_{1}\right) \int_{0}^{\infty} P_{n+1,1}(x) \mu_{1}(x) d x+\left(1-r_{2}\right) \int_{0}^{\infty} P_{n+1,2}(x) \mu_{2}(x) d x\right]
$$




$$
\begin{gathered}
+(1-q) \xi_{1}\left[\int_{0}^{\infty} R_{n+1,1}(x) \mu_{1}(x) d x+\int_{0}^{\infty} R_{n+1,2}(x) \mu_{2}(x) d x\right]+\xi_{1} \int_{0}^{\infty} W_{n}(x) \phi(x) d x+\lambda a_{1} \xi_{1} c_{n} E, n \geq 1 \\
P_{n, 2}(0)=(1-q) \xi_{2}\left[\left(1-r_{1}\right) \int_{0}^{\infty} P_{n+1,1}(x) \mu_{1}(x) d x+\left(1-r_{2}\right) \int_{0}^{\infty} P_{n+1,2}(x) \mu_{2}(x) d x\right] \\
+(1-q) \xi_{2}\left[\int_{0}^{\infty} R_{n+1,1}(x) \mu_{1}(x) d x+\int_{0}^{\infty} R_{n+1,2}(x) \mu_{2}(x) d x\right]+\xi_{2} \int_{0}^{\infty} W_{n}(x) \phi(x) d x+\lambda a_{1} \xi_{2} c_{n} E, n \geq 1 \\
R_{n, 1}(0)=r_{1} \int_{0}^{\infty} P_{n, 1}(x) \mu_{1}(x) d x \\
W_{n}(0)=q\left[\left(1-r_{1}\right) \int_{0}^{\infty} P_{n+1,1}(x) \mu_{1}(x) d x+\left(1-r_{2}\right) \int_{0}^{\infty} P_{n+1,2}(x) \mu_{2}(x) d x\right]
\end{gathered}
$$

and the normalizing condition

$$
E+\sum_{j=1}^{2} \sum_{n=1}^{\infty} \int_{0}^{\infty} P_{n, j}(x) d x+\sum_{j=1}^{2} \sum_{n=1}^{\infty} \int_{0}^{\infty} R_{n, j}(x) d x+\sum_{n=0}^{\infty} \int_{0}^{\infty} W_{n}(x) d x=1
$$

\section{Queue Size Distribution at Random Epoch}

Now let us multiply Equations (9)-(13) by $z^{n}$, and taking summations over all possible values of $n$ and simplifying we get

$$
\begin{aligned}
& \frac{d}{d x} P_{1}(x, z)+\left\{a_{1}(\lambda-\lambda C(z))+\mu_{1}(x)\right\} P_{1}(x, z)=0 \\
& \frac{d}{d x} P_{2}(x, z)+\left\{a_{1}(\lambda-\lambda C(z))+\mu_{2}(x)\right\} P_{2}(x, z)=0 \\
& \frac{d}{d x} R_{1}(x, z)+\left\{a_{1}(\lambda-\lambda C(z))+\mu_{1}(x)\right\} R_{1}(x, z)=0 \\
& \frac{d}{d x} R_{2}(x, z)+\left\{a_{1}(\lambda-\lambda C(z))+\mu_{2}(x)\right\} R_{2}(x, z)=0 \\
& \frac{d}{d x} W(x, z)+\left\{a_{2}(\lambda-\lambda C(z))+\phi(x)\right\} W(x, z)=0
\end{aligned}
$$

We now integrate Equations (22)-(26) between limits 0 and $x$ and obtain

$$
\begin{gathered}
P_{1}(x, z)=P_{1}(0, z) e^{-a_{1} \lambda(1-C(z))-\int_{0}^{x} \mu_{1}(t) d t} \\
P_{2}(x, z)=P_{2}(0, z) e^{-a_{1} \lambda(1-C(z))-\int_{0}^{x} \mu_{2}(t) d t} \\
R_{1}(x, z)=R_{1}(0, z) e^{-a_{1} \lambda(1-C(z))-\int_{0}^{x} \mu_{1}(t) d t} \\
R_{2}(x, z)=R_{2}(0, z) e^{-a_{1} \lambda(1-C(z))-\int_{0}^{x} \mu_{2}(t) d t} \\
W(x, z)=W(0, z) e^{-a_{2} \lambda(1-C(z))-\int_{0}^{x} \phi(t) d t}, \lambda>0
\end{gathered}
$$

Next we multiply Equation (16) with appropriate powers of $z$, take summation over all possible values of $n$ and using relations (15) and (27), after simplifying we obtain

$$
\begin{aligned}
z P_{1}(0, z)= & (1-q) \xi_{1}\left[\left(1-r_{1}\right) \int_{0}^{\infty} P_{1}(x, z) \mu_{1}(x) d x+\left(1-r_{2}\right) \int_{0}^{\infty} P_{2}(x, z) \mu_{2}(x, z) d x\right] \\
& +(1-q) \xi_{1}\left[\int_{0}^{\infty} R_{1}(x, z) \mu_{1}(x) d x+\int_{0}^{\infty} R_{2}(x, z) \mu_{2}(x) d x\right] \\
& +z \xi_{1} \int_{0}^{\infty} W(x, z) \phi(x) d x+z a_{1} \xi_{1} \lambda(C(z)-1) E
\end{aligned}
$$


We perform the same operations on Equations (17)-(20) and thus obtain

$$
\begin{gathered}
z P_{2}(0, z)=(1-q) \xi_{2}\left[\left(1-r_{1}\right) \int_{0}^{\infty} P_{1}(x, z) \mu_{1}(x) d x+\left(1-r_{2}\right) \int_{0}^{\infty} P_{2}(x, z) \mu_{2}(x, z) d x\right] \\
+(1-q) \xi_{2}\left[\int_{0}^{\infty} R_{1}(x, z) \mu_{1}(x) d x+\int_{0}^{\infty} R_{2}(x, z) \mu_{2}(x) d x\right] \\
+z \xi_{2} \int_{0}^{\infty} W(x, z) \phi(x) d x+z a_{1} \xi_{2} \lambda(C(z)-1) E \\
R_{n, 1}(0)=r_{1} \int_{0}^{\infty} P_{1}(x, z) \mu_{1}(x) d x \\
R_{n, 2}(0)=r_{2} \int_{0}^{\infty} P_{2}(x, z) \mu_{2}(x) d x \\
z W(0, z)=q\left[\left(1-r_{1}\right) \int_{0}^{\infty} P_{1}(x, z) \mu_{1}(x) d x+\left(1-r_{2}\right) \int_{0}^{\infty} P_{2}(x, z) \mu_{2}(x) d x\right]
\end{gathered}
$$

We now multiply Equations (27) and (29) by $\mu_{1}(x)$ and Equations (28) and (30) by $\mu_{2}(x)$, integrate by parts w.r.t. $x$, use Equation (2) to obtain

$$
\begin{aligned}
& \int_{0}^{\infty} P_{1}(x, z) \mu_{1}(x) d x=P_{1}(0, z) H_{1}^{*}\left(a_{1}(\lambda-\lambda C(z))\right) \\
& \int_{0}^{\infty} P_{2}(x, z) \mu_{2}(x) d x=P_{2}(0, z) H_{2}^{*}\left(a_{1}(\lambda-\lambda C(z))\right) \\
& \int_{0}^{\infty} R_{1}(x, z) \mu_{1}(x) d x=R_{1}(0, z) H_{1}^{*}\left(a_{1}(\lambda-\lambda C(z))\right) \\
& \int_{0}^{\infty} R_{2}(x, z) \mu_{2}(x) d x=R_{2}(0, z) H_{2}^{*}\left(a_{1}(\lambda-\lambda C(z))\right)
\end{aligned}
$$

Similarly multiplying Equation (31) by $\phi(x)$ and applying the same operations as above, we get

$$
\int_{0}^{\infty} W(x, z) \phi(x) d x=W(0, z) V^{*}\left(a_{2}(\lambda-\lambda C(z))\right)
$$

where $H_{j}^{*}\left(a_{1}(\lambda-\lambda C(z))\right)=\int_{0}^{\infty} e^{-a_{1}(\lambda-\lambda C(z)} d H_{j}(x)$ is the Laplace-Transform of $j$ th type of service, $j=1,2$ and $V^{*}\left(a_{2}(\lambda-\lambda C(z))\right)=\int_{0}^{\infty} e^{-a_{2}(\lambda-\lambda C(z))} d V(x)$ is the Laplace-Transform of vacation time.

Let us substitute relations (37)-(41) in Equations (32) and (33), thus we get

$$
\begin{aligned}
z P_{1}(0, z) & =(1-q) \xi_{1}\left[\left(1-r_{1}\right) P_{1}(0, z) H_{1}^{*}\left(a_{1}(\lambda-\lambda C(z))\right)+\left(1-r_{2}\right) P_{2}(0, z) H_{2}^{*}\left(a_{1}(\lambda-\lambda C(z))\right)\right]+ \\
& +(1-q) \xi_{1}\left[R_{1}(0, z) H_{1}^{*}\left(a_{1}(\lambda-\lambda C(z))\right)+R_{2}(0, z) H_{2}^{*}\left(a_{1}(\lambda-\lambda C(z))\right)\right]+z \xi_{1} W(0, z) V^{*}\left(a_{2}(\lambda-\lambda C(z))\right) \\
& \left.+z \lambda a_{1} \xi_{1}(C(z)-1)\right) E
\end{aligned}
$$

or

$$
\begin{aligned}
z P_{1}(0, z) & =(1-q) \xi_{1}\left[\left(1-r_{1}\right) P_{1}(0, z) H_{1}^{*}\left(a_{1}(\lambda-\lambda C(z))\right)+\left(1-r_{2}\right) P_{2}(0, z) H_{2}^{*}\left(a_{1}(\lambda-\lambda C(z))\right)\right] \\
& +(1-q) \xi_{1}\left[P _ { 1 } ( 0 , z ) \left(H_{1}^{*}\left(a_{1}(\lambda-\lambda C(z))\right)^{2}+P_{2}(0, z)\left(H_{2}^{*}\left(a_{1}(\lambda-\lambda C(z))\right)^{2}\right]+\right.\right. \\
& \left.z \xi_{1} q\left[\left(1-r_{1}\right) P_{1}(0, z) H_{1}^{*}\left(a_{1}(\lambda-\lambda C(z))\right)+\left(1-r_{2}\right) P_{2}(0, z) H_{2}^{*}\left(a_{1}(\lambda-\lambda C(z))\right)\right)\right] V^{*}\left(a_{2}(\lambda-\lambda C(z))\right) \\
& +z \lambda a_{1} \xi_{1}(C(z)-1) E
\end{aligned}
$$


Similarly

$$
\begin{aligned}
& z P_{2}(0, z)=(1-q) \xi_{2}\left[\left(1-r_{1}\right) P_{1}(0, z) H_{1}^{*}\left(a_{1}(\lambda-\lambda C(z))\right)+\left(1-r_{2}\right) P_{2}(0, z) H_{2}^{*}\left(a_{1}(\lambda-\lambda C(z))\right)\right] \\
& +(1-q) \xi_{2}\left[P _ { 1 } ( 0 , z ) \left(H_{1}^{*}\left(a_{1}(\lambda-\lambda C(z))\right)^{2}+P_{2}(0, z)\left(H_{2}^{*}\left(a_{1}(\lambda-\lambda C(z))\right)^{2}\right]+\right.\right. \\
& z \xi_{2}\left[\left(1-r_{1}\right) P_{1}(0, z) H_{1}^{*}\left(a_{1}(\lambda-\lambda C(z))\right)+\left(1-r_{2}\right) P_{2}(0, z) H_{2}^{*}\left(a_{1}(\lambda-\lambda C(z))\right)\right] V^{*}\left(a_{2}(\lambda-\lambda C(z))\right) \\
& +z \lambda a_{1} \xi_{2}(C(z)-1) E \begin{array}{r}
R_{1}(0, z)=r_{1} P_{1}(0, z) H_{1}^{*}\left(a_{1}(\lambda-\lambda C(z))\right) \\
R_{2}(0, z)=r_{2} P_{1}(0, z) H_{2}^{*}\left(a_{1}(\lambda-\lambda C(z))\right) \\
z W(0, z)=q\left[P_{1}(0, z) H_{1}^{*}\left(a_{1}(\lambda-\lambda C(z))\right)+P_{2}(0, z) H_{2}^{*}\left(a_{1}(\lambda-\lambda C(z))\right)\right]
\end{array}
\end{aligned}
$$

Now solving (42) and (43) we get the following

$$
\begin{gathered}
P_{1}(0, z)=\frac{z \lambda a_{1} \xi_{1}(1-C(z)) E}{D(z)} \\
P_{2}(0, z)=\frac{z \lambda a_{1} \xi_{2}(1-C(z)) E}{D(z)} \\
R_{1}(0, z)=\frac{z \lambda a_{1} r_{1} \xi_{1}(1-C(z)) H_{1}^{*}\left(a_{1}(\lambda-\lambda C(z))\right) E}{D(z)} \\
R_{2}(0, z)=\frac{z \lambda a_{1} r_{2} \xi_{2}(1-C(z)) H_{2}^{*}\left(a_{1}(\lambda-\lambda C(z) E\right.}{D(z)} \\
W(0, z)=\frac{q \lambda a_{1}\left[\left(1-r_{1}\right) \xi_{1} H_{1}^{*}\left(a_{1}(\lambda-\lambda C(z))\right)+\left(1-r_{2}\right) \xi_{2} H_{2}^{*}\left(a_{1}(\lambda-\lambda C(z))\right)\right] E}{D(z)}
\end{gathered}
$$

where $D(z)$ is given by the following relation

$$
\begin{aligned}
D(z)= & {\left[\left\{(1-q)+q V^{*}\left(a_{2}(\lambda-\lambda C(z))\right)\right\}\left\{\left(1-r_{1}\right) \xi_{1} H_{1}^{*}\left(a_{1}(\lambda-\lambda C(z))\right)+\left(1-r_{2}\right) \xi_{2} H_{2}^{*}\left(a_{1}(\lambda-\lambda C(z))\right)\right\}\right] } \\
& +(1-q)\left\{r _ { 1 } \xi _ { 1 } \left(H_{1}^{*}\left(a_{1}(\lambda-\lambda C(z))\right)^{2}+r_{2} \xi_{2}\left(H_{2}^{*}\left(a_{2}(\lambda-\lambda C(z))\right)^{2}\right\}-z\right.\right.
\end{aligned}
$$

Further we integrate Equations (27)-(31) w.r.t $x$ and use Equation (2) to obtain

$$
\begin{gathered}
P_{1}(z)=\frac{z \xi_{1}\left[1-H_{1}^{*}\left(a_{1}(\lambda-\lambda C(z))\right)\right] E}{D(z)} \\
P_{2}(z)=\frac{z \xi_{2}\left[1-H_{2}^{*}\left(a_{1}(\lambda-\lambda C(z))\right)\right] E}{D(z)} \\
R_{1}(z)=\frac{z r_{1} \xi_{1} H_{1}^{*}\left(a _ { 1 } ( \lambda - \lambda C ( z ) ) \left[1-H_{1}^{*}\left(a_{1}(\lambda-\lambda C(z))\right] E\right.\right.}{D(z)} \\
R_{2}(z)=\frac{z r_{2} \xi_{2} H_{2}^{*}\left(a _ { 1 } ( \lambda - \lambda C ( z ) ) \left[1-H_{2}^{*}\left(a_{1}(\lambda-\lambda C(z))\right] E\right.\right.}{D(z)} \\
W(z)=\frac{q \frac{a_{1}}{a_{2}}\left[1-V^{*}\left(a_{2}(\lambda-\lambda C(z))\right]\left[( 1 - r _ { 1 } ) \xi _ { 1 } H _ { 1 } ^ { * } \left(a_{1}(\lambda-\lambda C(z))+\left(1-r_{2}\right) \xi_{2} H_{2}^{*}\left(a_{1}(\lambda-\lambda C(z))\right] E\right.\right.\right.}{D(z)}
\end{gathered}
$$

Let us now define $P_{Q}(z)$ as the probability generating function of the queue size irrespective of the type of service the server is providing, such that adding Equations (52)-(56) we get

$$
P_{Q}(z)=P_{1}(z)+P_{2}(z)+R_{1}(z)+R_{2}(z)+W(z)
$$


We determine the unknown probability $E$ by using the relation of the normalizing condition

$$
E+P_{1}(1)+P_{2}(1)+R_{1}(1)+R_{2}(1)+W(1)=1
$$

We now use L'Hopital's Rule as the Equations (52)-(56) is indeterminate of the zero/zero form at $z=1$ and simplifying we obtain

$$
\begin{aligned}
& P_{1}(1)=\lim _{z \rightarrow 1} P_{1}(z) \\
& =\frac{\xi_{1} a_{1} \lambda E(I) E\left(S_{1}\right) E}{1-a_{1} \lambda E(I)\left\{\left(1-r_{1}\right) \xi_{1} E\left(S_{1}\right)+\left(1-r_{2}\right) \xi_{2} E\left(S_{2}\right)\right\}+q a_{2} \lambda E(I) E(V)\left\{\left(1-r_{1}\right) \xi_{1}+\left(1-r_{2}\right) \xi_{2}\right\}} \\
& P_{2}(1)=\lim _{z \rightarrow 1} P_{2}(z) \\
& =\frac{\xi_{2} a_{1} \lambda E(I) E\left(S_{2}\right) E}{1-a_{1} \lambda E(I)\left\{\left(1-r_{1}\right) \xi_{1} E\left(S_{1}\right)+\left(1-r_{2}\right) \xi_{2} E\left(S_{2}\right)\right\}+q a_{2} \lambda E(I) E(V)\left\{\left(1-r_{1}\right) \xi_{1}+\left(1-r_{2}\right) \xi_{2}\right\}} \\
& -2(1-q) a_{1} \lambda E(I)\left\{r_{1} \xi_{1} E\left(S_{1}\right)+r_{2} \xi_{2} E\left(S_{2}\right)\right\} \\
& R_{1}(1)=\lim _{z \rightarrow 1} R_{1}(z) \\
& =\frac{r_{1} \xi_{1} a_{1} \lambda E(I) E\left(S_{1}\right) E}{1-a_{1} \lambda E(I)\left\{\left(1-r_{1}\right) \xi_{1} E\left(S_{1}\right)+\left(1-r_{2}\right) \xi_{2} E\left(S_{2}\right)\right\}+q a_{2} \lambda E(I) E(V)\left\{\left(1-r_{1}\right) \xi_{1}+\left(1-r_{2}\right) \xi_{2}\right\}} \\
& -2(1-q) a_{1} \lambda E(I)\left\{r_{1} \xi_{1} E\left(S_{1}\right)+r_{2} \xi_{2} E\left(S_{2}\right)\right\} \\
& R_{2}(1)=\lim _{z \rightarrow 1} R_{2}(z) \\
& =\frac{r_{2} \xi_{2} a_{1} \lambda E(I) E\left(S_{2}\right) E}{1-a_{1} \lambda E(I)\left\{\left(1-r_{1}\right) \xi_{1} E\left(S_{1}\right)+\left(1-r_{2}\right) \xi_{2} E\left(S_{2}\right)\right\}+q a_{2} \lambda E(I) E(V)\left\{\left(1-r_{1}\right) \xi_{1}+\left(1-r_{2}\right) \xi_{2}\right\}} \\
& -2(1-q) a_{1} \lambda E(I)\left\{r_{1} \xi_{1} E\left(S_{1}\right)+r_{2} \xi_{2} E\left(S_{2}\right)\right\} \\
& W(1)=\lim _{z \rightarrow 1} W(z) \\
& =\frac{q a_{1} \lambda E(I) E(V)\left\{\left(1-r_{1}\right) \xi_{1}+\left(1-r_{2}\right) \xi_{2}\right\} E}{\left.1-a_{1} \lambda E(I)\left(1-r_{1}\right) \xi_{1} E\left(S_{1}\right)+\left(1-r_{2}\right) \xi_{2} E\left(S_{2}\right)\right\}+q a_{2} \lambda E(I) E(V)\left\{\left(1-r_{1}\right) \xi_{1}+\left(1-r_{2}\right) \xi_{2}\right\}} \\
& -2(1-q) a_{1} \lambda E(I)\left\{r_{1} \xi_{1} E\left(S_{1}\right)+r_{2} \xi_{2} E\left(S_{2}\right)\right\}
\end{aligned}
$$

where $E(I)$ is the mean size of batch of arriving customers, $E\left(S_{1}\right), E\left(S_{2}\right)$ are the mean service times of type 1 and type 2 server respectively, $E(V)$ is the mean of vacation time and $H^{*}(0)=1, V^{*}(0)=1$.

The RHS of the results (58)-(62) respectively give the steady state probability that the server is busy providing type 1 service, type 2 services, repeating service of type 1, repeating type 2 service and server being on vacation.

Now adding Equations (58)-(62) we get

$$
\begin{gathered}
P_{Q}(1)=\frac{\left[a_{1} \lambda E(I)\left\{\left(1+r_{1}\right) \xi_{1} E\left(S_{1}\right)+\left(1+r_{2}\right) \xi_{2} E\left(S_{2}\right)\right\}+q a_{1} \lambda E(I) E(V)\left\{\left(1-r_{1}\right) \xi_{1}+\left(1-r_{2}\right) \xi_{2}\right\}\right] E}{1-a_{1} \lambda E(I)\left\{\left(1-r_{1}\right) \xi_{1} E\left(S_{1}\right)+\left(1-r_{2}\right) \xi_{2} E\left(S_{2}\right)\right\}+q a_{2} \lambda E(I) E(V)\left\{\left(1-r_{1}\right) \xi_{1}+\left(1-r_{2}\right) \xi_{2}\right\}} \\
-2(1-q) a_{1} \lambda E(I)\left\{r_{1} \xi_{1} E\left(S_{1}\right)+r_{2} \xi_{2} E\left(S_{2}\right)\right\}
\end{gathered}
$$

which gives the steady state probability that the server is busy, irrespective of whether it is providing type 1 or type 2 service and server being on vacation.

Let us simplify the normalizing condition $E+P_{Q}(1)=1$ to get $\mathrm{E}$ and thus we have

$$
E=\frac{\begin{array}{r}
1-a_{1} \lambda E(I)\left\{\left(1-r_{1}\right) \xi_{1} E\left(S_{1}\right)+\left(1-r_{2}\right) \xi_{2} E\left(S_{2}\right)\right\}-q a_{2} \lambda E(I) E(V)\left\{\left(1-r_{1}\right) \xi_{1}+\left(1-r_{2}\right) \xi_{2}\right\} \\
-2(1-q) a_{1} \lambda E(I)\left\{r_{1} \xi_{1} E\left(S_{1}\right)+r_{2} \xi_{2} E\left(S_{2}\right)\right\}
\end{array}}{1+q a_{1} \lambda E(I) E(V)\left\{\left(1-r_{1}\right) \xi_{1}+\left(1-r_{2}\right) \xi_{2}\right\}-q a_{2} \lambda E(I) E(V)\left\{\left(1-r_{1}\right) \xi_{1}+\left(1-r_{2}\right) \xi_{2}\right\}}
$$

Also from (64) we obtain $\rho$ as the utilization factor of the system as $\rho=1-E$. 
Now let $P_{S}(z)$ denote the probability generating function of the system size irrespective of the state of the system

$$
P_{S}(z)=P_{1}(z)+P_{2}(z)+R_{1}(z)+R_{2}(z)+W(z)+E
$$

Thus

$$
P_{S}(z)=\frac{E\left[\begin{array}{c}
\left\{(1-q)+q V^{*}\left(a_{2}(\lambda-\lambda C(z))+q \frac{a_{1}}{a_{2}}\left[1-V^{*}\left(a_{2}(\lambda-\lambda C(z))\right]-z\right\}\left\{\left(1-r_{1}\right) \xi_{1} H_{1}^{*}\left(a_{1}(\lambda-\lambda C(z))\right)+\right.\right.\right. \\
\left.\left(1-r_{2}\right) \xi_{2} H_{2}^{*}\left(a_{1}(\lambda-\lambda C(z))\right)\right\}+\{(1-q)-z\}\left\{r _ { 1 } \xi _ { 1 } \left(H_{1}^{*}\left(a_{1}(\lambda-\lambda C(z))\right)^{2}+r_{2} \xi_{2}\left(H_{2}^{*}\left(a_{1}(\lambda-\lambda C(z))\right)^{2}\right\}\right.\right.
\end{array}\right]}{\left[\left\{(1-q)+q V^{*}\left(a_{2}(\lambda-\lambda C(z))\right\}\left\{( 1 - r _ { 1 } ) \xi _ { 1 } H _ { 1 } ^ { * } \left(a_{1}(\lambda-\lambda C(z))+\left(1-r_{2}\right) \xi_{2} H_{2}^{*}\left(a_{1}(\lambda-\lambda C(z))\right\}\right.\right.\right.\right.}+\left(\begin{array}{c}
(1-q)\left\{r_{1} \xi_{1}\left(H_{1}^{*}\left(a_{1}(\lambda-\lambda C(z))\right)^{2}+r_{2} \xi_{2}\left(H_{2}^{*}\left(a_{1}(\lambda-\lambda C(z))\right)^{2}\right\}\right]-z\right.
\end{array}\right.
$$

\section{The Average Queue Size}

Let $L q$ denote the mean queue size at random epoch. Then

$$
L_{q}=\lim _{z \rightarrow 1} \frac{d}{d z} P_{Q}(z)
$$

Since this formula is also of zero/zero form, we use L'Hopital's Rule twice and obtain

$$
L_{S}=\lim _{z \rightarrow 1} \frac{D^{\prime}(z) N^{\prime \prime}(z)-N^{\prime}(z) D^{\prime \prime}(z)}{2\left(D^{\prime}(z)\right)^{2}}
$$

where primes and double primes in (66) denote first and second derivatives at $\mathrm{z}=1$ respectively.

$$
\begin{gathered}
N^{\prime}(1)=-E_{0}\left[\begin{array}{c}
a_{1} \lambda E(I)\left\{\left(1+r_{1}\right) \xi_{1} E\left(S_{1}\right)+\left(1+r_{2}\right) \xi_{2} E\left(S_{2}\right)\right\} \\
-q a_{1} \lambda E(I) E(V)\left\{\left(1-r_{1}\right) \xi_{1}+\left(1-r_{2}\right) \xi_{2}\right\}
\end{array}\right] \\
N^{\prime \prime}(1)=-E\left[\begin{array}{l} 
\\
\left.2 a_{1} \lambda E(I)\left\{\left(1-r_{1}\right) \xi_{1} E\left(S_{1}\right)+\left(1-r_{2}\right) \xi_{2} E\left(S_{2}\right)\right\}+a_{1} \lambda E(I / I-1)\left\{\xi_{1} E\left(S_{1}\right)+\xi_{2} E\left(S_{2}\right)\right\}\right) \\
+a_{1} \lambda E(I)\left\{r_{1} \xi_{1} E\left(S_{1}\right)+r_{2} \xi_{2} E\left(S_{2}\right)\right\}+2\left(a_{1} \lambda E(I)\right)^{2}\left\{r_{1} \xi_{1} E\left(S_{1}^{2}\right)+r_{2} \xi_{2} E\left(S_{2}^{2}\right)\right\} \\
+\left(a_{1} \lambda E(I / I-1)\right)\left\{r_{1} \xi_{1} E\left(S_{1}\right)+r_{2} \xi_{2} E\left(S_{2}\right)\right\}-\left(a_{1} \lambda E(I)\right)^{2}\left\{r_{1} \xi_{1}\left(E\left(S_{1}\right)\right)^{2}+r_{2} \xi_{2}\left(E\left(S_{2}\right)\right)^{2}\right\} \\
+2 q(a \lambda E(I))^{2} E(V)\left\{\left(1-r_{1}\right) \xi_{1} E\left(S_{1}\right)+\left(1-r_{2}\right) \xi_{2} E\left(S_{2}\right)\right\} \\
+\left\{q\left(a_{1} \lambda E(I)\right)^{2} E\left(V^{2}\right)+a_{1} \lambda E(I / I-1) E(V)\right\}\left\{\left(1-r_{1}\right) \xi_{1}+\left(1-r_{2}\right) \xi_{2}\right\} \\
D^{\prime}(1)= \\
a_{1} \lambda E(I)\left[\left(1-r_{1}\right) \xi_{1} E\left(S_{1}\right)+\left(1-r_{2}\right) \xi_{2} E\left(S_{2}\right)\right]+q a_{2} \lambda E(I) E(V)\left\{\left(1-r_{1}\right) \xi_{1}+\left(1-r_{2}\right) \xi_{2}\right\}
\end{array}\right] \\
D^{\prime \prime}(1)=\left[\begin{array}{l}
\left\{\left(a_{1} \lambda E(I)\right)^{2}\left\{\left(1-r_{1}\right) \xi_{1} E\left(S_{1}^{2}\right)+\left(1-r_{2}\right) \xi_{2} E\left(S_{2}^{2}\right)\right]\right. \\
\left.\left.a_{1} \lambda E(I / I-1)\right\}\left(1-r_{1}\right) \xi_{1} E\left(S_{1}\right)+\left(1-r_{2}\right) \xi_{2} E\left(S_{2}\right)\right\}+a_{1} a_{2}(\lambda E(I))^{2}\left\{\left(1-r_{1}\right) \xi_{1} E\left(S_{1}\right)+\left(1-r_{2}\right) \xi_{2} E\left(S_{2}\right)\right\} \\
+q\left(a_{2} \lambda E(I)\right)^{2} E\left(V^{2}\right)\left\{\left(1-r_{1}\right) \xi_{1}+\left(1-r_{2}\right) \xi_{2}\right\}+2(1-q)\left[( a _ { 1 } \lambda E ( I ) ) ^ { 2 } \left\{r_{1} \xi_{1} E\left(S_{1}^{2}\right)+r_{2} \xi_{2} E\left(S_{2}^{2}\right)\right.\right. \\
\left.+a_{1} \lambda E(I / I-1)\left\{r_{1} \xi_{1} E\left(S_{1}\right)+r_{2} \xi_{2} E\left(S_{2}\right)\right\}+\left(a_{1} \lambda E(I)\right)^{2}\left\{r_{1} \xi_{1}\left(E\left(S_{1}\right)\right)^{2}+r_{2} \xi_{2}\left(E\left(S_{2}\right)\right)^{2}\right\}\right]
\end{array}\right]
\end{gathered}
$$

where $E\left(S_{1}^{2}\right), E\left(S_{2}^{2}\right), E\left(V^{2}\right)$ are the second moments of service times of type 1, type 2 and vacation time respectively. $E(I / I-1)$ is the second factorial moment of the batch of arriving customers, $H_{1}^{*}(0)=1, H_{2}^{*}(0)=1$, $V^{*}(0)=1$ and $E$ has been obtained in (64).

We can also obtain $L=L_{q}+\rho$ by using the above relations, which gives the steady-state average size of the queue system. 


\section{Particular Cases}

Case 1. Re-service in a Vacation queue with Batch arrival and Two types of General Heterogeneous Service. Here we consider the situation when there is no balking i.e. all customers join the system, and in that case, we take $a_{1}=a_{2}=1$; thus Equation (65) becomes

$$
\begin{gathered}
E(1-z)\left\{\left(1-r_{1}\right) \xi_{1} H_{1}^{*}(\lambda-\lambda C(z))+\left(1-r_{2}\right) \xi_{2} H_{2}^{*}(\lambda-\lambda C(z))\right\} \\
P_{S}(z)=\frac{+\{(1-q)-z\}\left\{r_{1} \xi_{1}\left(H_{1}^{*}(\lambda-\lambda C(z))\right)^{2}+r_{2} \xi_{2}\left(H_{2}^{*}(\lambda-\lambda C(z))\right)^{2}\right\}}{\left\{(1-q)+q V^{*}(\lambda-\lambda C(z))\right\}\left\{\left(1-r_{1}\right) \xi_{1} H_{1}^{*}(\lambda-\lambda C(z))+\left(1-r_{2}\right) \xi_{2} H_{2}^{*}(\lambda-\lambda C(z))\right\}} \\
+(1-q)\left\{r_{1} \xi_{1}\left(H_{1}^{*}(\lambda-\lambda C(z))\right)^{2}+r_{2} \xi_{2}\left(H_{2}^{*}(\lambda-\lambda C(z))\right)^{2}\right\}
\end{gathered}
$$

is the queue size distribution of a batch arrival queuing system with two types of general heterogeneous service with vacation and optional re-service where idle time $E$

$$
E=\frac{-q \lambda E(I) E(V)\left\{\left(1-r_{1}\right) \xi_{1}+\left(1-r_{2}\right) \xi_{2}\right\}+2 q \lambda E(I)\left\{r_{1} \xi_{1} E\left(S_{1}\right)+r_{2} \xi_{2} E\left(S_{2}\right)\right\}}{1-2(1-q) \lambda E(I)\left\{r_{1} \xi_{1} E\left(S_{1}\right)+r_{2} \xi_{2} E\left(S_{2}\right)\right\}}
$$

Case 2. Balking and Re-service with Batch arrival with and two types of general Heterogeneous Service. Here we assume that the server does not take vacation, i.e. $q=0$; and obtain

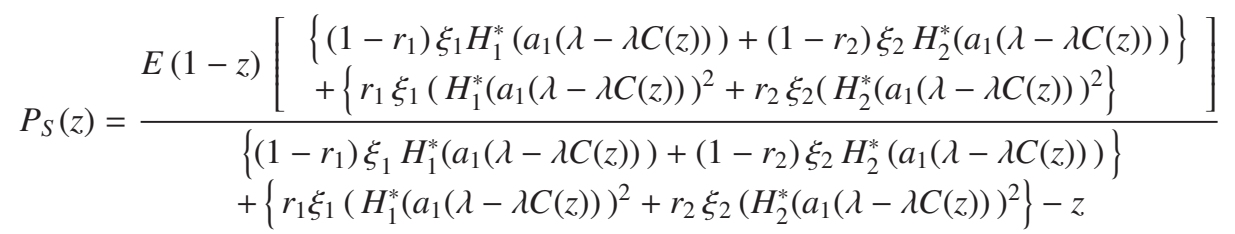

is the queue size distribution of Batch arrival queuing system with two types of general heterogeneous service with balking and optional re-service and

$$
E=\frac{1-a_{1} \lambda E(I)\left\{\xi_{1} E\left(S_{1}\right)+\xi_{2} E\left(S_{2}\right)\right\}-a_{1} \lambda E(I)\left\{r_{1} \xi_{1} E\left(S_{1}\right)+r_{2} \xi_{2} E\left(S_{2}\right)\right\}}{1-2 a_{1} \lambda E(I)\left\{r_{1} \xi_{1} E\left(S_{1}\right)+r_{2} \xi_{2} E\left(S_{2}\right)\right\}}
$$

Case 3. Balking in a Vacation queue with batch arrival and two types of Heterogeneous service. Here we assume that there is no re-service, then $r_{1}=r_{2}=0$, our queue size distribution reduces to

$$
\begin{gathered}
P_{S}(z)=\frac{E\left[\left\{(1-q)+q V^{*}\left(a_{2}(\lambda-\lambda C(z))\right)+q \frac{a_{1}}{a_{2}}\left[1-V^{*}\left(a_{2}(\lambda-\lambda C(z))\right)\right]-z\right\}\left\{\xi_{1} H_{1}^{*}\left(a_{1}(\lambda-\lambda C(z))\right)+\xi_{2} H_{2}^{*}\left(a_{1}(\lambda-\lambda C(z))\right)\right\}\right]}{\left[\left\{(1-q)+q V^{*}\left(a_{2}(\lambda-\lambda C(z))\right)\right\}\left\{\xi_{1} H_{1}^{*}\left(a_{1}(\lambda-\lambda C(z))\right)+\xi_{2} H_{2}^{*}\left(a_{1}(\lambda-\lambda C(z))\right)\right\}\right]-z} \\
E=\frac{1-a_{1} \lambda E(I)\left\{\xi_{1} E\left(S_{1}\right)+\xi_{2} E\left(S_{2}\right)\right\}-q a_{2} \lambda E(I) E(V)}{1+q a_{1} \lambda E(I) E(V)-q a_{2} \lambda E(I) E(V)} .
\end{gathered}
$$

Case 4. If we assume that there is two types of heterogeneous service, optional re-service, no vacation, no balking, then $q=0 ; a_{1}=a_{2}=1$, the PGF in (65) reduces to

$$
\begin{aligned}
P_{S}(z)=\frac{E\left[\begin{array}{l}
\{1-z\}\left\{\left(1-r_{1}\right) \xi_{1} H_{1}^{*}(\lambda-\lambda C(z))+\left(1-r_{2}\right) \xi_{2} H_{2}^{*}(\lambda-\lambda C(z))\right\} \\
+(1-z)\left\{r_{1} \xi_{1}\left(H_{1}^{*}(\lambda-\lambda C(z))\right)^{2}+r_{2} \xi_{2}\left(H_{2}^{*}(\lambda-\lambda C(z))\right)^{2}\right\}
\end{array}\right]}{\left[\begin{array}{l}
\left\{\left(1-r_{1}\right) \xi_{1} H_{1}^{*}(\lambda-\lambda C(z))+\left(1-r_{2}\right) \xi_{2} H_{2}^{*}(\lambda-\lambda C(z))\right\} \\
\left\{r_{1} \xi_{1}\left(H_{1}^{*}(\lambda-\lambda C(z))\right)^{2}+r_{2} \xi_{2}\left(H_{2}^{*}(\lambda-\lambda C(z))\right)^{2}\right.
\end{array}\right]-z} \\
\left.E=1-\lambda E(I)\left\{\left(1+r_{1}\right) \xi_{1} E\left(S_{1}\right)+\left(1+r_{2}\right) \xi_{2} E\left(S_{2}\right)\right)\right\}
\end{aligned}
$$

Which tallies with the result obtained by Madan et al. (2004) for a $M^{X} /\left(\begin{array}{l}G_{1} \\ G_{2}\end{array}\right) 1$ queue with optional re-service.

Case 5. Again if we consider the situation when there is no case of optional re-service and no balking (i.e. all arriving batches join the system) with two types of heterogeneous service and vacation then,$r_{1}=r_{2}=0$; $a_{1}=a_{2}=1$, then (65) becomes

$$
P_{S}(z)=\frac{E\left[(1-z)\left\{\xi_{1} H_{1}^{*}(\lambda-\lambda C(z))+\xi_{2} H_{2}^{*}(\lambda-\lambda C(z))\right\}\right]}{\left[\left\{(1-q)+q V^{*}(\lambda-\lambda C(z)\}\left\{\xi_{1} H_{1}^{*}\left(\lambda-\lambda C(z)+\xi_{2} H_{2}^{*}(\lambda-\lambda C(z)\}\right]-z\right.\right.\right.}
$$




$$
E=1-\lambda E(I)\left\{\xi_{1} E\left(S_{1}\right)+\xi_{2} E\left(S_{2}\right)\right\}-q \lambda E(I) E(V)
$$

Thus our result (71) is equivalent to the result obtained by Madan et al. (2005) for the PGF of a queue size distribution at random epoch of $M^{X} /\left(\begin{array}{c}G_{1} \\ G_{2}\end{array}\right) / 1 / G(B S) / V_{S}$ with batch arrivals, two kinds of general heterogeneous service and general vacation time with Bernoulli Schedules.

Case 6. Now further we assume that in addition to the particular cases in (5), if we take $\xi_{2}=0$; i.e. $\xi_{1}=1$, which means the server is providing only one type of service and without re-service and balking, we obtain

$$
\begin{gathered}
P_{S}(z)=\frac{E(1-z) H_{1}^{*}(\lambda-\lambda C(z))}{\{(1-q)+q V *(\lambda-\lambda C(z))\} H_{1}^{*}(\lambda-\lambda C(z))-z} \\
E=1-\lambda E(I) \xi_{1} E\left(S_{1}\right)-q \lambda E(I) E(V)
\end{gathered}
$$

Thus the result (72) reduces to the probability generating function (PGF) of a queue size distribution at a random epoch for $M^{X} / G / 1 / G(B S) / V_{S}$ queue with batch arrivals, general service and general vacation first studied by Madan (2004) who considered first service general and second service exponential.

Case 7. Now if we consider a system with two types of general heterogeneous service but no optional re-service, no vacation and no balking, i.e. $r_{1}=r_{2}=0, q=0, a_{1}=a_{2}=1$, then we obtain the queue size

$$
\begin{gathered}
P_{S}(z)=\frac{E(1-z)\left\{\xi_{1} H_{1}^{*}(\lambda-\lambda C(z))+\xi_{2} H_{2}^{*}(\lambda-\lambda C(z))\right\}}{\left\{\xi_{1} H_{1}^{*}\left(\lambda-\lambda C(z)+\xi_{2} H_{2}^{*}(\lambda-\lambda C(z))\right\}-z\right.} \\
E=1-\lambda E(I) \xi_{1} E\left(S_{1}\right)-\lambda E(I) \xi_{2} E\left(S_{2}\right)
\end{gathered}
$$

Case 8. Again in addition to particular cases in (73), if we assume that there is only one type of service then

$$
\begin{aligned}
P_{S}(z) & =\frac{E(1-z) H_{1}^{*}(\lambda-\lambda C(z))}{H_{1}^{*}(\lambda-\lambda C(z))-z} \\
E & =1-\lambda E(I) \xi_{1} E\left(S_{1}\right)
\end{aligned}
$$

is the queue size distribution of a $M^{X} / G / 1$ batch arrival providing single service follows the result obtained by Gaver (1959).

\section{References}

Baba, Y. (1986). On the $M^{X} / G / 1$ queue with vacation time. Operation Research Letters, 5, 93-98. http://dx.doi.org/10.1016/0167-6377(86)90110-0

Borthakur, A., \& Choudhury, G. (1997). On a batch arrival queue with generalized Vacation. Sankhya-Series B, 59, 369-383.

Choudhury, G. (2002). Analysis of the $M^{X} / G / 1$ queuing system with vacation times. Sankhya-Series B, 64(1), $37-49$.

Choudhury, G., \& Madan, K. C. (2007). A batch arrival Bernoulli vacation queue with random set up time under restricted admissibility policy. International Journal of Operation Research (USA), 2(1), 81-97. http://dx.doi.org/10.1504/IJOR.2007.011445

Doshi, B. T. (1986). Queuing Systems with Vacation - a survey. Queuing Systems 1, 29-66. http://dx.doi.org/10.1007/BF01149327

Gaver, D. P. (1959). Imbedded markov chain analysis of a waiting line process in continuous time. Ann. Mathematical Statistics, 30, 698-720. http://dx.doi.org/10.1214/aoms/1177706200

Glenbe, G., \& Pujolle, G. (1987). Introduction to Queueing Networks. New York: John Wiley and Sons.

Lee, H. W., \& Lee, S. S. (1993). Corrected distribution of idle period in $M / G / 1$ queue with multiple vacations. Research Report, Department of Industrial Engineering, Sung Kyon University, Suwon, Korea.

Jeyakumar, S., \& Arumuganathan. (2011). A Non-Markovian Queue with Multiple Vacations and Control Policy on Request for Re-service. Quality Technology of Quantitative Management (QTQM), 8(3), 253-269. 
Madan, K. C. (1988). A cyclic queuing system with three servers and optional two-way feedback. Microelectron Rel., 28(6), 873-875. http://dx.doi.org/10.1016/0026-2714(88)90285-5

Madan, K. C. (2000). An $M / G / 1$ queue with second optional service. Queuing Systems Theory Appl., 34, 1-4, 37-46.

Madan, K. C., \& Abu-Dayyeh, W. (2002). Restricted admissibility of batches into an $M / G / 1$ type bulk queue with modified Bernoulli schedule server vacations. ESAIM Probability Statistics, 6, 113-125. http://dx.doi.org/10.1051/ps:2002006

Madan, K. C., \& Choudhury, G. (2004). Steady state analysis of an $M^{X} /\left(G_{1} G_{2}\right) / 1$ queue with restricted admissibility and modified Bernoulli schedule server vacation. Journal of Probability and Statistical Science, 2(2), 167-185.

Madan, K. C., Al-Rawi, Z. R., \& Al-Nasser, A. D. (2005). On a $\left.M^{X} / G_{1} G_{2}\right) / 1 / G(B S) / V_{s}$ vacation queue with two types of general heterogeneous service. Journal of Applied Mathematics and Decision Sciences, 3, 123-135. http://dx.doi.org/10.1155/JAMDS.2005.123

Takagi, H. (1990). Time-dependent analysis of an $M / G / 1$ vacation models with exhaustive service. Queueuing Systems, 6(1), 369-390. http://dx.doi.org/10.1007/BF02411484 